\title{
Early-phase Neutrophilia in Cigarette Smoke-induced Acute Eosinophilic Pneumonia
}

\author{
Keisuke MIKI, Mari MIKI*, Yoichi NAKAMURA*, Yoshihiro SuZUKI**, Yoshio OKAnO*, \\ Fumitaka OGUSHI*, Yuji OHTSUKI*** and Takuro NAKAYAMA
}

\begin{abstract}
Although cigarette smoking is a recognized cause of acute eosinophilic pneumonia (AEP), and an increase in eosinophils in the lung is a common occurrence in AEP, early-phase neutrophilia in AEP is not well understood. We describe three cases of cigarette smoke (menthol type)-induced AEP with neutrophilia in the lungs or blood. Increased in-vitro production of the neutrophil chemoattractant interleukin (IL)-8 by human bronchial epithelial cells (HBECs) was correlated with neutrophilia. We suggest that $\mathrm{IL-8}$ released from HBECs is involved in neutrophilia in the lung in AEP, and is newly recognized as an important factor in the early phase of AEP development.
\end{abstract}

(Internal Medicine 42: 839-845, 2003)

Key words: cigarette smoke extract, interleukin 8, bronchial epithelial cell, neutrophilia, acute eosinophilic pneumonia

\section{Introduction}

Acute eosinophilic pneumonia (AEP) was first described in 1989 as a unique entity of eosinophilic lung disease characterized by acute respiratory failure, diffuse pulmonary infiltrates and massive eosinophilia in bronchoalveolar lavage (BAL) fluid (BALF) (1). Since then, many factors such as drugs, fungi, and environmental agents including cigarette smoke have been implicated as causes of $\operatorname{AEP}(2,3)$. The number of reports describing cigarette smoke-induced AEP (CS-AEP) is increasing and several of these have demonstrated eosinophils to be the major cell population contributing to inflammation associated with $\operatorname{CS}-\operatorname{AEP}(4,5)$. Interest- ingly, neutrophilia in the lung or blood is usually found during the early phase of CS-AEP $(4,5)$, although the precise mechanism remains to be elucidated. Herein we examine three cases of CS-AEP, one case of which was included in our previous report (6).

\section{Case Report}

\section{Case 1}

An 18-year-old women started smoking several cigarettes $\left(\mathrm{HOPE}^{\circledR}\right)$ per day from January 2000 , and stopped smoking 1 month later. Two weeks prior to admission on June 15, 2000 , she had resumed smoking 7 or 8 cigarettes per day but with a change in brand $\left(\right.$ Marlboro menthol $\left.{ }^{\oplus}\right)$. She was admitted to our hospital, with a sudden history of dry cough, chest pain, high fever $\left(39^{\circ} \mathrm{C}\right)$, and dyspnea worsening since June 14. No medication had been taken before the onset of symptoms. She had no previous history of asthma or atopic disease. On admission, the physical examination revealed a respiratory rate of 32 breaths per minute. Cardiac examination was within normal limits. While the patient was breathing room air, the arterial blood gas values were $\mathrm{pH}, 7.469$; $\mathrm{Paco}_{2}, 35.0 \mathrm{mmHg}$; and $\mathrm{PaO}_{2}, 46.8 \mathrm{mmHg}$. The $\mathrm{WBC}$ count was $16,200 / \mu$ with $88 \%$ neutrophils, $5 \%$ lymphocytes, and $7 \%$ monocytes, without an increase in eosinophils. Chest computed tomography (CT) scans revealed diffuse bilateral pulmonary infiltrates (Fig. 1A). Since the above findings were not alleviated by treatment with antibiotics (clarithromycin, mionomycin, and meropenem trihydrate) from admission, she underwent steroid pulse therapy from day 3 post-admission methylprednisolone sodium succinate, 1,000 $\mathrm{mg} /$ day $\times 3$ days followed by fiberoptic bronchoscopy on day 6 post-admission (the 7th day from onset of symptoms). A BALF sample included an abundance of leukocytes with $3 \%$ neutrophils, $48 \%$ lymphocytes, $20 \%$ macrophages, $2 \%$ basophils, and $27 \%$ eosinophils that had nuclear hyper-

From the Department of Internal Medicine, JA Kochi Hospital, Nankoku, *the Clinical Research Center, Kochi National Hospital, Kochi, **the Department of Internal Medicine, Hyogo Prefectural Awaji Hospital, Sumoto and ***the Second Department of Pathology, Kochi Medical School, Nankoku Received for publication December 9, 2002; Accepted for publication April 25, 2003

Reprint requests should be addressed to Dr. Keisuke Miki, the Department of Internal Medicine, JA Kochi Hospital, 526-1 Myoken-aza-Nakano, Nankoku, Kochi 783-8509 


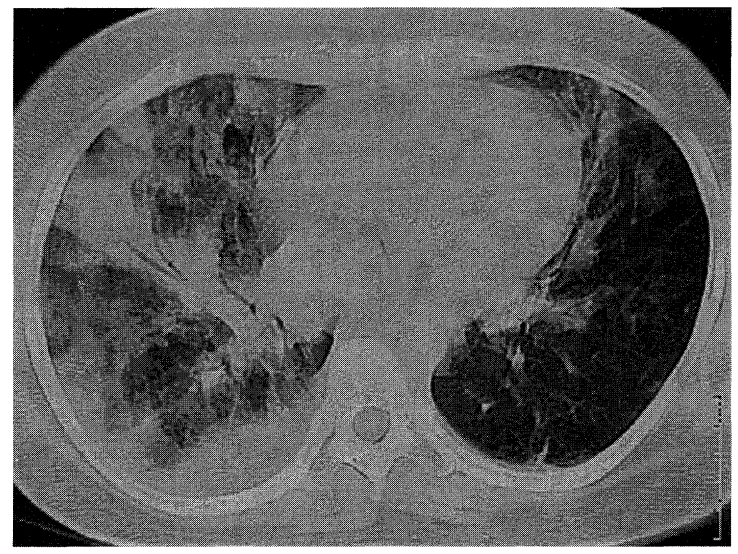

A

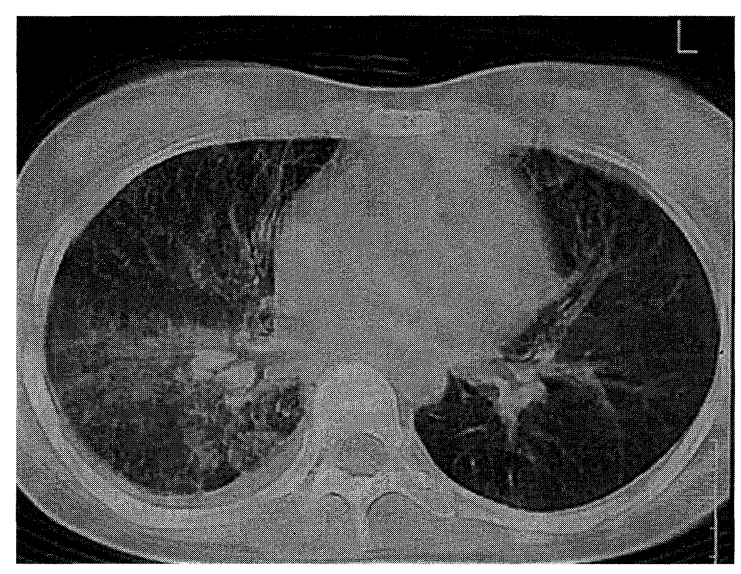

B

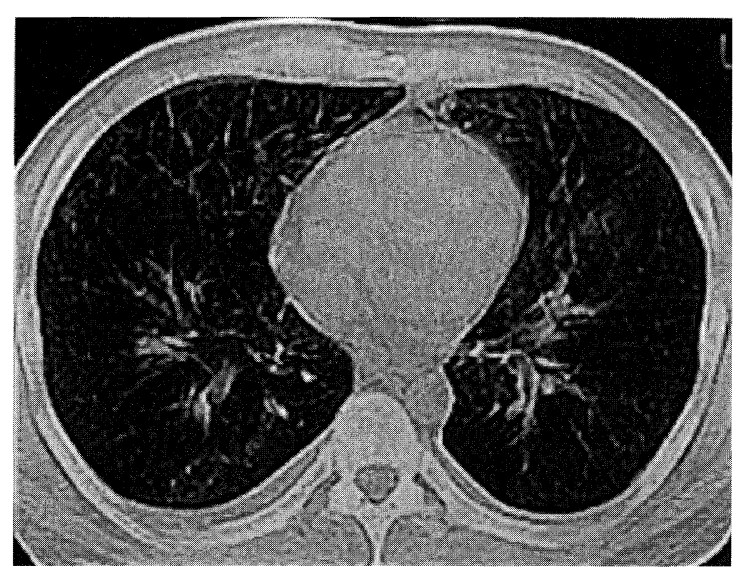

C

Figure 1. A: case 1, B: case 2, C: case 3. In case 1 and 2, chest computed tomography scans on admission, showing diffuse patchy infiltrates with pleural effusion, predominantly around bronchi in lower fields of the lung, but in case 3 only thickening of bronchial walls with pleural effusion. segmentation (three nuclei, 29\%; four nuclei, 10\%). Immunophenotypic analysis of BALF lymphocytes gave a CD4/CD8 ratio of 1.79 . On day 3 post-admission (the 4 th day from onset of symptoms), the WBC count was $9,800 / \mu \mathrm{l}$ with $76 \%$ neutrophils, $12 \%$ lymphocytes, $3 \%$ monocytes, and $9 \%$ eosinophils. IL-5 and IL-8 levels in peripheral blood were $854 \mathrm{pg} / \mathrm{ml}$ and $22 \mathrm{pg} / \mathrm{ml}$, respectively. Oral prednisolone, $30 \mathrm{mg} / \mathrm{day}$, was initiated on day 6 postadmission, followed by clinical improvement and decline in neutrophilia and eosinophilia. A subsequent chest X-ray film revealed clearing of pulmonary infiltrates. From the clinical course, this patient met the diagnostic criteria of AEP. Prednisolone was tapered off for 19 days, and she was discharged from the hospital on day 23 .

\section{Case 2}

A 16-year-old woman started smoking 5 or 6 cigarettes $\left(\mathrm{HOPE}^{\circledR}\right)$ per day 1 mo. prior to admission and changed to another brand (Marlboro menthol ${ }^{\circledR}$ ) 3 weeks before admission. On October 23, 2000, she experienced sudden chest pain and fever $\left(39^{\circ} \mathrm{C}\right)$, and was admitted to our hospital on October 25. No medication had been taken before the onset of the symptoms. There were no unusual findings in her past medical histories. Chest CT scans demonstrated patchy infiltrates predominantly in the lower field of both lungs with prominence around the bronchi (Fig. 1B). The following laboratory data were obtained the 3rd day from onset of symptoms: Arterial blood gas analysis; and $\mathrm{Pao}_{2}, 64.8 \mathrm{mmHg}$ with oxygen by nasal cannula at $5 \mathrm{l} / \mathrm{min}$. Peripheral blood examination showed a WBC count of $15,000 / \mu \mathrm{l}$, and a differential cell count of $92 \%$ neutrophils, $4 \%$ lymphocytes, and $4 \%$ monocytes without eosinophils. IL-5 and IL-8 levels in peripheral blood were $1,831 \mathrm{pg} / \mathrm{ml}$ and $24 \mathrm{pg} / \mathrm{ml}$, respectively. A BALF sample obtained on day 2 post-admission (the 4th day from onset of symptoms) included an abundance of leukocytes with 5\% neutrophils, 52\% lymphocytes, $18 \%$ macrophages, and $25 \%$ eosinophils with nuclear hypersegmentation (three nuclei, 13\%). Immunophenotypic analysis of BALF lymphocytes showed a CD4/CD8 ratio of 2.15. Steroid pulse therapy was initiated on day 2 post-admission (methylprednisolone sodium succinate, $500 \mathrm{mg} /$ day $\times 3$ days). On day 5 post-admission, the WBC count was $5,800 / \mu 1$ with $18 \%$ eosinophils $44 \%$ lymphocytes, $1 \%$ basophils, and $7 \%$ monocytes without neutrophilia (30\%), and chest X-ray film and IL-5 level in peripheral blood showed marked improvement. Lymphocyte-stimulating tests using cigarettes $\left(\mathrm{HOPE}^{\circledR}\right.$ and Marlboro menthol ${ }^{\oplus}$ ) were negative. After discharge, the patient resumed smoking cigarettes other than Marlboro menthol ${ }^{\oplus}$ without any symptoms.

\section{Case 3}

A 26-year-old man was in good health until admission day, June 28, 2001, when he noted fever, chest pain, and progressive shortness of breath. He had started to smoke 10 cigarettes (Marlboro menthol ${ }^{\boxplus}$ ) per day 2 weeks before admission. No medications had been taken prior to this illness. 
On admission (the 1st day from onset of symptoms), the physical examination revealed a respiratory rate of 30 breaths per minute. Cardiac examination was normal. While the patient was breathing room air, the arterial blood gas values were pH, 7.414; $\mathrm{Paco}_{2}, 36.7 \mathrm{mmHg} ; \mathrm{PaO}_{2}, 48.3 \mathrm{mmHg}$. The WBC count was $16,800 / \mu$ l with $88 \%$ neutrophils, $4 \%$ lymphocytes, and 7\% monocytes without eosinophilia (1\%). IL-5 and IL-8 levels in peripheral blood were $3,531 \mathrm{pg} / \mathrm{ml}$ and $158 \mathrm{pg} / \mathrm{ml}$, respectively. Chest X-ray and chest CT (Fig. 1C) showed thickening of bronchial walls, predominantly in the lower field of both lungs, without patchy infiltrates. Twelve hours after admission, a transbronchial lung biopsy (TBLB) specimen showed neutrophil infiltration in the alveolar septa, rather than eosinophil. Steroid pulse therapy was started on day 2 (methylprednisolone sodium succinate, $500 \mathrm{mg} /$ day $\times 3$ days) just before fiberoptic bronchoscopic examination. On day 2, soon after examination of fiberoptic bronchoscopy, Chest CT scan demonstrated patchy infiltrates clearly in the lower field of the lung (data not shown). Oral prednisolone, $30 \mathrm{mg} /$ day, was initiated on day 5 . On day 7 , the WBC count was $9,800 / \mu$ l with $27 \%$ lymphocytes, $5 \%$ monocytes, and $21 \%$ eosinophils, followed by a decline, without neutrophilia (47\%), and chest X-ray film and level of IL-5 and IL-8 in peripheral blood showed marked improvement. Prednisolone was tapered off for 15 days and he was discharged home on day 17.

\section{Transbronchial Lung Biopsies}

Pulmonary tissues obtained by TBLB, were fixed in $10 \%$ buffered formalin and then embedded in paraffin in the usual manner. Four micrometer dewaxed sections were stained with hematoxylin eosin (HE) and direct fast scarlet (DFS) in order to disclose eosinophils. Then, immunohistochemical staining was also performed. Pancytokeratin antibodies (AE1/AE3, $1: 400$, DAKO, Kyoto, Japan) for the pulmonary epithelium, CD68 (KP-1, 1:100, DAKO) for alveolar macrophages and $\alpha$-smooth muscle actin $(1: 200$, DAKO) for myofibroblasts were used to investigate these tissues by the avidin-biotin peroxidase complex (ABC) method. Both AE1/AE3 and CD68 antibodies needed $0.1 \%$ pronase pretreatment for antigen retrieval.

Pulmonary tissues from cases 1 and 2 by TBLB revealed peribronchial and perivascular marked eosinophil infiltration with edema, intermingling lymphocytes, macrophages, and plasma cells (Fig. 2A, TBLB specimen in case 2 not shown). These inflammatory cells were also detected in alveolar septa and spaces, and veins (Fig. 2A). In case 1, but not case 2, fibrin exudation widely occurred in alveolar spaces (Fig. $2 \mathrm{~A}$ ), revealing no organizing processes, although intraalveolar fibrin lumps were occasionally covered by a thin alveolar epithelium, which was positive for pancytokeratin antibodies (Fig. 2B). Alveolar macrophages were seen to be aggregated or scattered in alveolar spaces in parts. By DFS stain, orange-colored eosinophils were observed very clearly (Fig. 2C). These histopathological changes in case 1 were the most conspicuous of our three cases. In cases 1 and 3, but not in case 2, TBLB specimens showed neutrophil infiltration. In case 3, neutrophil infiltration, rather than eosinophil infiltration, was predominant (Fig. 2D).

\section{Diagnosis}

In all patients, the results of serum mycoplasma, chlamydia, aspergillus, and viral antibody tests and bacterial examinations were all negative. Cases 1and 2 were diagnosed as AEP, consistent with the diagnostic criteria of Allen and Davis (7). Since in case 3 BAL was not performed due to severe hypoxemia during examination, a diagnosis of AEP was made from the clinical course, laboratory data, and TBLB findings, consistent with the modified diagnostic criteria of AEP (2).

\section{Effect of Exposure to Cigarette Smoke on Human Bronchial Epithelial Cells}

After obtaining informed consent, HBECs were obtained from a healthy non-smoking volunteer, and from cases 2 and 3 , two months after discharge from the hospital. The collection and culture of HBECs were performed as previously described $(6,8)$. First, 10 brushings (Model BC-15C, Olympus) to collect HBECs were performed from the subsegmental bronchi with 10 gentle strokes on the epithelial surface via bronchoscopy. The cells obtained by brushing were collected in a 50-ml polypropylene tube containing $20 \mathrm{ml}$ RPMI 1640 with gentamicin by shaking the brush in the medium. The cells were then washed twice by centrifugation at $400 \times g$ at $4{ }^{\circ} \mathrm{C}$ for 10 minutes. The rinsed pellet of cells obtained by brushing was resuspended in LHC9/RPMI 1640 medium. We plated aliquots of HBECs in LHC9/RPMI 1640 medium on 24-well culture plates, and incubated the plates until the cells had grown to confluency (primary culture). The medium was changed after 24 hours and then every 48 hours. The BEAS-2B cell line (human bronchial epithelial cell line) was purchased from the American Type Culture Collection (Manassas, VA) and cultured as recommended by the manufacturer. Then, HBECs and BEAS-2B cells were exposed to cigarette smoke extract (CSE) as previously described (6). Briefly, CSE was generated by respectively burning a

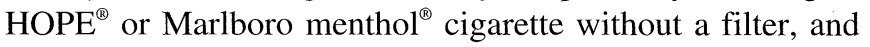
leading the smoke stream $(60 \mathrm{ml})$ into a syringe. The cigarette smoke was then repeatedly bubbled ten times through another syringe containing $20 \mathrm{ml}$ RPMI 1640. The resulting suspension was adjusted to $\mathrm{pH} 7.4$ with concentrated $\mathrm{NaOH}$ and filtered through a 0.45 - $\mu$ pore filter (Millipore Corp., Bedford, MA). HBECs and BEAS-2B cells were exposed to the medium containing the $10 \% \mathrm{CSE}$-suspension for 6 hours or 24 hours. After these exposures, the culture supernatants were measured with enzyme-linked immunosorbent assays (ELISAs) for interleukin (IL)-4, IL-8, vascular endothelial growth factor (VEGF), and granulocyte macrophage-colony stimulating factor (GM-CSF), as described previously (9). 


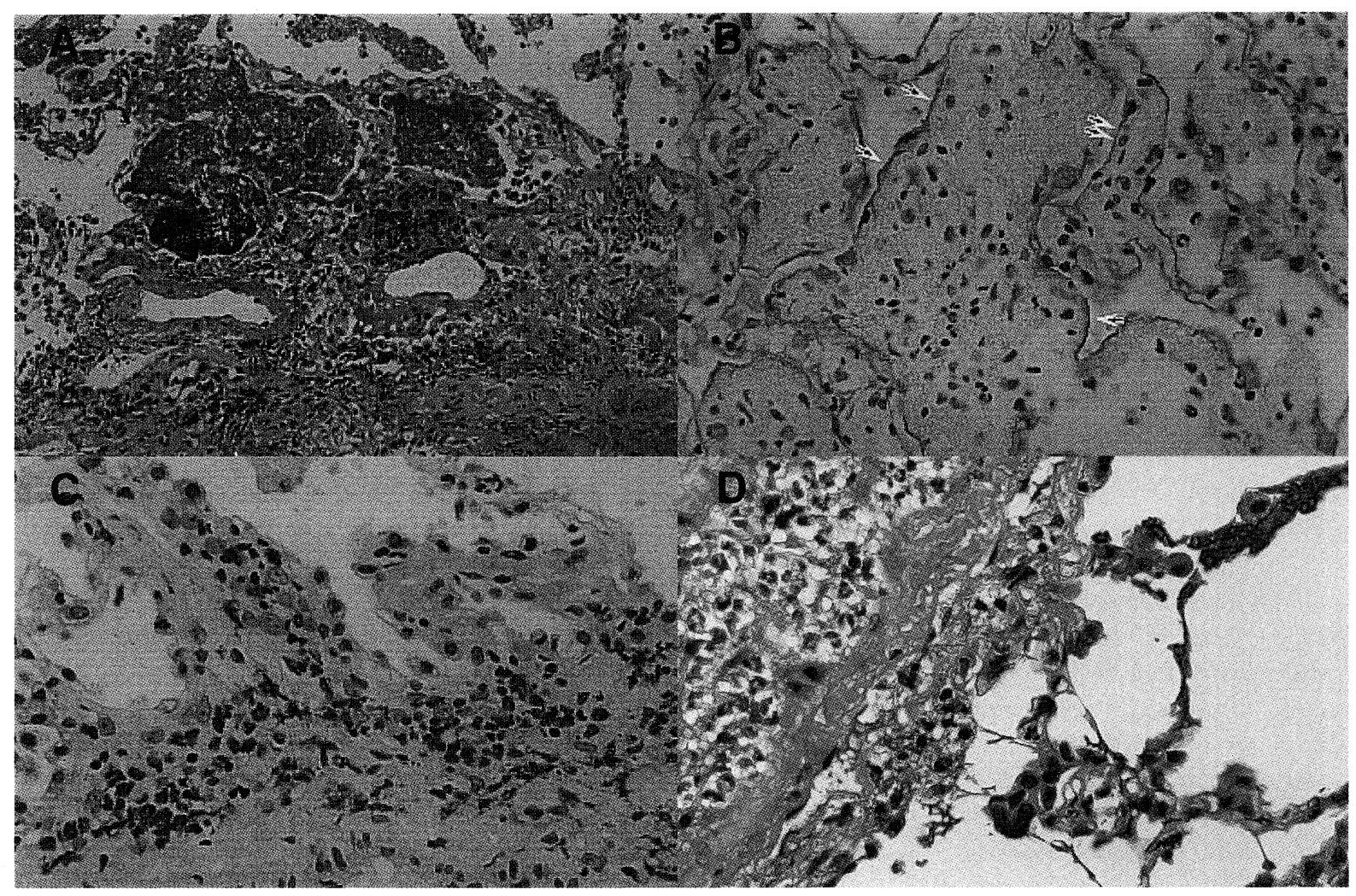

Figure 2. Transbronchial lung biopy specimens in case 1 after chest computed tomography scans showed patchy infiltrates in the lower field of the lung, but in case 3 just before patchy infiltrates were observed on chest computed tomography scans. A: Intraalveolar fibrin exudation and thickening of alveolar septa with eosinophil infiltration. Case 1, HE stain, $\times 220$. B: Thin pulmonary epithelium lining on fibrin clots (arrows). Note alveolar septa (double arrow). Case 1, pancytokeratin AE1/AE3 antibody stain, $\times 430$. C: Marked eosinophil infiltration, exhibiting orangecolored cytoplasm in the perivascular region and alveolar septa. Case 1, DFS stain, $\times 430$. D: Neutrophil infiltration, but not eosinophil infiltration, predominates, Case 3 , HE stain, $\times 430$.

Results showed that only the exposure of HBECs from case 2 (Fig. 3A, top panel) or 3 (Fig. 3A, middle panel) to CSE from Marlboro menthol ${ }^{\oplus}$ cigarettes resulted in increased production of IL-8 in a time-dependent manner, particularly in case 2. In contrast, exposure of HBECs (obtained from case 2) to CSE from $\mathrm{HOPE}^{\circledast}$ did not increase the production of IL-8 (Fig. 3A, top panel). The exposure of HBECs in the normal volunteer and BEAS-2B cells to CSE from either Marlboro menthol ${ }^{\circledast}$ or $\mathrm{HOPE}^{\oplus}$ led to no significant increase in release of IL-8 (Fig. 3 A, bottom panel and 3B). These findings indicated that the significant increase of IL-8 production induced by Marlboro menthol ${ }^{\oplus}$ was specific to AEP patients. In addition, 6 hours and 24 hours exposure of HBECs to CSE from either Marlboro menthol ${ }^{\circledast}$ or $\mathrm{HOPE}^{\circledast}$ had no influence on the production of IL-4, GM-CSF, or VEGF in cases 2 and 3 (data not shown).

Since endotoxin is known to stimulate IL- 8 production from HBECs, we examined whether the concentration of endotoxin affected the differential effect of CSE from 2 cigarettes on IL-8 production in this study. We measured endotoxin in the CSEs (HOPE ${ }^{\oplus}$ and Marlboro menthol ${ }^{\circledR}$ ) and
RPMI 1640 alone using a kinetic turbidimetric assay. The concentrations of endotoxin were as follows: $\mathrm{HOPE}^{\oplus}, 3.8 \pm$ $0.1 \mathrm{pg} / \mathrm{ml}$; Marlboro menthol ${ }^{\oplus}, 2.2 \pm 1.1 \mathrm{pg} / \mathrm{ml}$; only RPMI 1640 without CSE, $2.0 \pm 0.6 \mathrm{pg} / \mathrm{ml}(\mathrm{n}=2)$. From these data we concluded that the effect of the endotoxin was not important.

\section{Discussion}

Cigarette smoking has recently been recognized to cause AEP and in Japan some reports have demonstrated an association of CS-AEP with the Marlboro menthol ${ }^{\varpi}$ brand $(2,10$, 11). Herein, we described three cases of cigarette smokinginduced AEP accompanied by neutrophilia in the lung, which correlated with increased production of IL-8 from HBECs. This response both in-vivo and in-vitro is specific to the brand of cigarette (menthol type). Although cigarette smoke has the capacity to damage the bronchi in a number of ways, including direct toxicity to the bronchial epithelium, oxidative damage, and recruitment of inflammatory cells (12), the mechanisms by which the over 4,000 components of smoke (including menthol) (12) affect the incidence or 

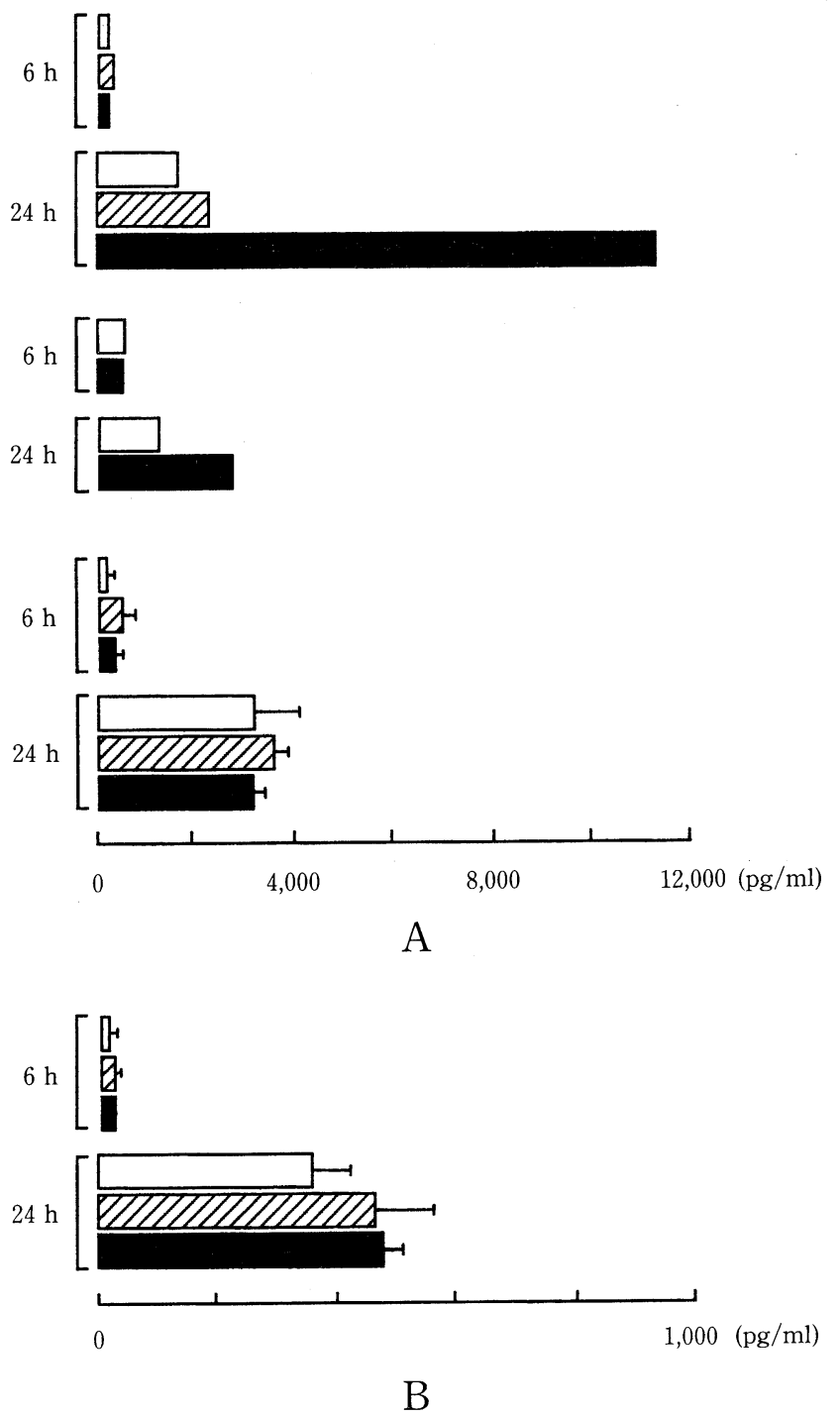

Figure 3. In vitro secretion of IL-8 by HBECs and BEAS-2B cells after $6 \mathrm{~h}$ or $24 \mathrm{~h}$ stimulation with the $10 \%$ CSE from Marlboro menthol ${ }^{\circledast}$ (closed bars), HOPE ${ }^{\circledR}$ (hatched bars) or no CSE (open bars). IL-8 concentration was determined by ELISA. A) Top panel: HBECs from case 2 (single experiment). Middle panel: HBECs from case 3 (single experiment). Bottom panel: HBECs from a normal volunteer. Data are means $\pm S E$ of triplicate experiments. B) BEAS-2B cells. Data are means \pm SE of triplicate experiments. There was no significant difference in IL-8 production by HBECs from a normal volunteer, or BEAS2B cells among CSE from Marlboro menthol ${ }^{\oplus}$, HOPE $^{\oplus}$, and no CSE (Tukey-Kramer's test).

severity of AEP are completely unknown. The evidence presented here allowed us to elucidate the triggering mechanism for AEP.

A noteworthy phenomenon is that an increase in neutrophils circulating in the blood occurs in the early phase of CSAEP and is followed by an increase in eosinophils circulating in the blood, as previously described $(3,4,6)$. In acute interstitial pneumonia such as hypersensitivity pneumonia (13), drug-induced eosinophilic pneumonia (EP) (14), and idiopathic AEP (3), evidence indicates that elevated neutrophils appear in the blood in the early or active phase of disease. Therefore, circulating neutrophilia may not be a phenomenon unique to CS-AEP. On the other hand, in the lung, neutrophilia appearing before eosinophilia seems to be important in the pathogenesis of CS-AEP.

In the early phase of AEP, a reverse correlation between the number of neutrophils and eosinophils in the BALF of one out of four patients was reported by Allen et al (1). More recently, Fujimura et al reported that neutrophils and lymphocytes, rather than eosinophils, in the lung may be related to the pathogenesis of AEP, comparing BALF cells among AEP, chronic eosinophilic pneumonia (CEP), and druginduced EP (15). In addition, there was no significant difference in the mean number of nuclear segmentation (16), which is associated with the disease activity of $\mathrm{EP}$, in eosinophils in BALF among patients with AEP, CEP, and drug-induced EP (15). In the present study, examination of nuclear segmentation in BALF, pulmonary tissues by TBLB, and clinical course from the 3 cases, revealed the most severe disease in case 1 , while the disease phase in case 3 was the earliest, as fiberoptic bronchoscopy was performed. In cases 1 and 2, the CD4/CD8 ratios of lymphocytes in BALF were high, which is consistent with the observation of Fujimura et al (15). It is possible that this increase in CD4/CD8 ratio suggests that activated helper T-cells are involved in the pathogenesis of CS-AEP. In case 3, a TBLB specimen showed neutrophil infiltration in the alveolar septa, rather than eosinophil infiltration, just before chest CT scans revealed patchy shadows, although neutrophil infiltration was not detected in case 2 and the number of neutrophils in BALF was not markedly elevated in case 1 or case 2 . This difference may be partially due to the time between appearance of symptoms and examination by fiberoptic bronchoscopy. These findings suggest that the degree of lung infiltration of neutrophils, as well as activated eosiniphils, is dependent on the clinical severity/phase in AEP patients and the possibility that early phase neutrophils in the lung have an impact on the pathogenesis of AEP. Moreover, this seems to be supported by the previous observation showing that a significant number of neutrophils, but not eosinophils, in the BALF of guinea pigs was found in only the early phase after single CS exposure followed by a decline. On the other hand, repeated cigarette smoke exposure clearly resulted in significant initial neutrophilia, with no further increase after that, followed by eosinophilia in the BALF of guinea pigs in the time course (17).

Since the airway epithelium is the first line of defense against cigarette smoke and roentgenographic infiltrates were observed predominantly around the bronchi in each case, the differential phenomenon of HBECs to CSE from those two cigarette types, especially Marlboro menthol ${ }^{\oplus}$, may explain the triggering mechanisms for AEP. The wellstudied neutrophil chemoattractant IL-8, and neutrophils are 
known to be important in airway inflammation associated with cigarette smoke (18-21). BALF specimens from nonasthmatic smokers have greater concentrations of IL-8 and neutrophils than non-smokers, while numbers of eosinophils are not elevated (18). Therefore, we examined the in vivo effects of cigarette smoke exposure and in vitro effects of exposure to CSE on production of soluble factors, such as IL-8, by HBECs. Although IL-8 levels in the peripheral blood are not particularly elevated, levels of IL-8 are remarkably high in culture supernatants of HBECs after exposure to Marlboro menthol ${ }^{\circledR}$ CSE in cases 2 and 3, indicating that increased IL- 8 was the result of a local immune response. In case 3 , we found a high concentration of IL-8 in the peripheral blood just before the appearance of patchy infiltrates on chest X-ray film. However, the levels of IL-8 in cases 1 and 2 were not elevated. Further, Nakahara et al reported that the increased IL-8 is a nonspecific change in AEP (22). This discrepancy may be partially due to differences in the time of IL- 8 production. This seems to be supported by a previous study which showed that IL-8 release by A549 epithelial cells in response to the thermophilic bacteria, but not cigarette smoke, started to increase after 12 hours and peaked at 48 hours (23), suggesting that IL-8 production plays a role in the initial immune response. We found the neutrophilia in the lung on the TBLB specimen in cases 1 and 3, but not case 2, and that IL- 8 levels in peripheral blood in cases 1 and 2 were not elevated, which seems to deny the relevance between circulating IL-8 and neutrophilia in the lung. However, this interpretation depends on time elapsed between the onset of the symptoms and the performance of fiberoptic bronchoscopy. Thus, the timing of observation is an important variable accounting for discrepant data.

IL-5-stimulated eosinophils respond to IL-8 because IL-8 receptors were undetectable on freshly isolated eosinophils, but were detectable after priming with IL-5 in vitro (24). In contrast, the addition of anti-IL- 8 antibody significantly enhanced chemotaxis of BALF eosinophils from AEP patients, suggesting an inhibitory effect of IL-8 on eosinophils (22). Moreover, a previous study indicated a significant correlation between IL-8 levels and the number of neutrophils, but not eosinophils, in the BALF of patients with EP (25). In the present paper, we showed in-vivo and in-vitro evidence that the effects of menthol-containing cigarette smoke on IL-8 production is a specific phenomenon. Therefore, it is possible that local production of IL-8 in the lung is more closely connected with the numbers of neutrophils than eosinophils in the early phase of AEP. In addition, these findings suggest that locally produced IL-8 from HBECs may play important roles in the pathophysiology of AEP by inducing neutrophilia in the lung in the early phase in AEP.

Several investigators have suggested that eosinophils represent the major cell population contributing to the inflammatory reaction associated with AEP (1-5). In the present cases, eosinophils with hypersegmented nuclei were clearly found in BALF. Furthermore, elevated IL-5, which was seen in all of our cases, may initiate the recruitment of eosino- phils, increase eosinophil survival, and enhance the release of mediators from eosinophils $(26,27)$. More recently, the level of eotaxin (25), a potent and eosinophil-specific chemoattractant, and thymus- and activation-regulated chemokine (TARC) (28), was found to be high in patients with EP; their level was significantly correlated with the eosinophil number in BALF. This suggests that eotaxin and TARC, as well as IL-5, play important roles in lung eosinophilia in patients with EP, although we did not measure those concentrations in this study.

Steroid therapy is known to have a dramatic effect on the function of eosinophils in vitro (29) and in patients with AEP (1-7). As well, IL-8 secretion from respiratory epithelial cells is suppressed by dexamethasone (23). In the present study, the disease activity in case 1 was the most severe, likely due to delayed start of steroid therapy. Although the efficacy of the steroid therapy has not been demonstrated in detail in the present study, the presence of severe hypoxemia leading to possible fatality leads us to administer steroid therapy as soon as possible.

In summary, after smoking the same brand of cigarette (Marlboro menthol ${ }^{\circledR}$ ) three young patients presented with AEP accompanied by neutrophilia in the lungs or blood. For young patients with AEP, we should pay attention to the smoking history, particularly to the use of menthol-type cigarettes. Although a wide range of factors is involved in the pathogenesis of CS-AEP and further analyses and larger studies are necessary to elucidate the role of IL-8 in AEP, we present the possibility that secretion of IL- 8 by HBECs is associated with an increased number of neutrophils in the lung, and that the release of IL- 8 by HBECs plays a role in the early phase of CS-AEP pathogenesis.

\section{References}

1) Allen JN, Pacht ER, Gadek JE, Davis WB. Acute eosinophilic pneumonia as a reversible cause of noninfectious respiratory failure. $\mathrm{N}$ Engl J Med 321: 569-574, 1989.

2) Nakajima M, Matsushima T. Acute eosinophilic pneumonia following cigarette smoking. Intern Med 39: 759-760, 2000.

3) Pope-Harman AL, Davis WB, Allen ED, Christoforidis AJ, Alllen JN. Acute eosinophilic pneumonia. A summary of 15 cases and review of the literature. Medicine 75: 334-342, 1996.

4) Shiota $Y$, Kawai T, Matsumoto H, et al. Acute eosinophilic pneumonia following cigarette smoking. Intern Med 39: 830-833, 2000.

5) Shintani H, Fujimura M, Yasui $M$, et al. Acute eosinophilic pneumonia caused by cigarette smoking. Intern Med 39: 66-68, 2000.

6) Miki K, Miki M, Okano Y, et al. Cigarette smoke-induced acute eosinophilic pneumonia accompanied with neutrophilia in the blood. Intern Med 41: 993-996, 2002.

7) Allen JN, Davis WB. Eosinophilic lung diseases. Am J Respir Crit Care Med 150: 1423-1438, 1994.

8) Nakamura Y, Azuma M, Okano Y, et al. Upregulatory effects of interleukin-4 and interleukin-13 but not interleukin-10 on granulocyte/ macrophage colony-stimulating factor production by human bronchial epithelial cells. Am J Respir Cell Mol Biol 15: 680-687, 1996.

9) Miki K, Shimizu E, Yano S, Tani K, Sone S. Demethylation by 5-aza$2^{\prime}$-deoxycytidine $\left(5\right.$-azadC) of $p 16^{I N K 4 A}$ gene results in downregulation of vascular endothelial growth factor expression in human lung cancer cell lines. Oncol Res 12: 335-342, 2000. 


\section{AEP with Neutrophilia in the Early Phase}

10) Matsui S, Yamashita N, Maruyama M, et al. Acute eosinophilic pneumonia induced by cigarette smoking: positive lymphocyte stimulating test of cigarette extract. J Jpn Respir Soc (Nihon Kokyuki Gakkai Zasshi) 38: 807-811, 2000 (in Japanese, Abstract in English).

11) Ito $S$, Taniguchi $H$, Kondo $Y$, et al. Positive response to smoking challenge test in a case of acute eosinophilic pneumonia. J Jpn Respir Soc (Nihon Kokyuki Gakkai Zasshi) 37: 424-428, 1999 (in Japanese, Abstract in English).

12) Rahman I, MacNee W. Oxidant/antioxidant imbalance in smokers and chronic obstructive pulmonary disease. Thorax 51: 348-350, 1996.

13) Kawai $T$, Tamura M, Murao M. Summer-type hypersensitivity pneumonitis. A unique diseasae in Japan. Chest 85: 311-317, 1984.

14) Yokoyama A, Mizushima $Y$, Suzuki H, Arai N, Kitagawa M, Yano S. Acute eosinophilic pneumonia induced by minocycline: prominent Kerley B lines as a feature of positive rechallenge test. Jpn J Med 29: 195-198, 1990.

15) Fujimura $M$, Yasui $M$, Shinagawa $S$, Nomura $M$, Matsuda $T$. Bronchoalveolar lavage cell findings in three types of eosinophilic pneumonia: acute, chronic and drug-induced eosinophilic pneumonia. Respir Med 92: 743-749, 1998.

16) Chihara J, Nakajima S. Induction of hypodense eosinophils and nuclear hypersegmentation of eosinophils by various chemotactic factors and lymphokines in vitro. Allergy Proc 10: 27-32, 1989.

17) Matsumoto $K$, Aizawa $H$, Inoue $H$, et al. Eosinophilic airway inflammation induced by repeated exposure to cigarette smoke. Eur Respir $\mathbf{J}$ 12: 387-394, 1998.

18) Kuschner WG, D'Alessandro A, Wong H, Blanc PD. Dose-dependent cigarette smoking-related inflammatory responses in healthy adults. Eur Respir J 9: 1989-1994, 1996.

19) Rusznak C, Mills PR, Devalia JL, Sapsford RJ, Davies RJ, Lozewicz S. Effect of cigarette smoke on the permeability and IL-1 $\beta$ and sICAM1 release from cultured human bronchial epithelial cells of neversmokers, smokers and patients with chronic obstructive pulmonary disease. Am J Respir Cell Mol Biol 23: 530-536, 2000.

20) Hinman LM, Stevens CA, Matthay RA, Gee JB. Elastase and lysozyme activities in human alveolar macrophages. Effects of cigarette smoking. Am Rev Respir Dis 121: 263-271, 1980.

21) Thompson AB, Huerta G, Robbins RA, et al. The bronchitis index. A semiquantitative visual scale for the assessment of airways inflammation. Chest 103: 1482-1488, 1993.

22) Nakahara Y, Hayashi S, Fukuno Y, Kawashima M. Yatsunami J. Increased interleukin-5 levels in bronchoalveolar lavage fluid is a major factor for eosinophil accumulation in acute eosinophilic pneumonia. Respiration 68: 389-395, 2001.

23) Gudmundsson G, Hunninghake GW. Respiratory epithelial cells release interleukin- 8 in response to a thermophilic bacteria that causes hypersensitivity pneumonitis. Exp Lung Res 25: 217-228, 1999.

24) Heath H, Qin S, Rao P, et al. Chemokine receptor usage by human eosinophils. The importance of CCR3 demonstrated using an antagonistic monoclonal antibody. J Clin Invest 99: 178-184, 1997.

25) Katoh S, Matsumoto N, Fukushima K, et al. Elevated chemokine levels in bronchoalveolar lavage fluid of patients with eosinophilic pneumonia. J Allergy Clin Immunol 106: 730-736, 2000.

26) Lopez AF, Sanderson CJ, Gamble JR, Campbell HD, Young IG, Vadas MA. Recombinant human interleukin 5 is a selective activator of human eosinophil function. J Exp Med 167: 219-224, 1988.

27) Wang JM, Rambaldi A, Biondi A, Chen ZG, Sanderson CJ, Mantovani A. Recombinant human interleukin 5 is a selective eosinophil chemoattractant. Eur J Immunol 19: 701-705, 1989.

28) Miyazaki E, Nureki S, Fukami T, et al. Elevated levels of thymus- and activation-regulated chemokine in bronchalveolar lavage fluid from patients with eosinophilic pneumonia. Am J Respir Crit Care Med 165: 1125-1131, 2002.

29) Altman LC, Hill JS, Hairfield WM, Mullarkey MF. Effects of corticosteroids on eosinophil chemotaxis and adherence. J Clin Invest 67: 28-36, 1981. 